The Israeli Journal of Aquaculture - Bamidgeh, IJA_69.2017.1415, 8 pages

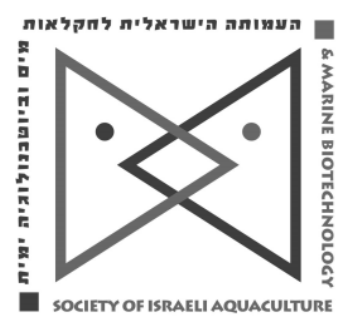

The IJA appears exclusively as a peer-reviewed on-line open-access journal at http://www.siamb.org.il. To read papers free of charge, please register online at registration form.

Sale of $I J A$ papers is strictly forbidden.

\title{
Dietary Probiotic Bacillus licheniformis TC22 Increases Growth, Immunity, and Disease Resistance, against Vibrio splendidus Infection in Juvenile Sea Cucumbers Apostichopus japonicus
}

\author{
Yancui Zhao ${ }^{1, *}$, Lei Yuan ${ }^{2}$, Mingzhu Li ${ }^{3}$, Junli Wan ${ }^{1}$, Yiyan Wang ${ }^{1}$, Hushan Sun ${ }^{1}$ \\ ${ }^{1}$ School of Life Science, Ludong University, 186 Hongqi Middle Road, Yantai 264025, P.R. \\ China \\ 2 Department of Food Engineering, Shandong Business Institute, Yantai 264670, P.R. China. \\ ${ }^{3}$ School of agriculture, Ludong University, 186 Hongqi Middle Road, Yantai 264025, P.R. China
}

Keywords: Apostichopus japonicus; probiotic; growth; immunity; Bacillus

\begin{abstract}
In this study we examined the effects of probiotic Bacillus licheniformis TC22 on growth, immunity, and disease resistance against Vibrio splendidus in juvenile sea cucumbers Apostichopus japonicus. For 30 days, sea cucumbers were fed diets with TC22 at 0 (control), $10^{5}, 10^{7}$, and $10^{9} \mathrm{CFU} / \mathrm{g}$ respectively. Results showed that dietary TC22 at $10^{9} \mathrm{CFU} / \mathrm{g}$ significantly improved $(P<0.05)$ growth of sea cucumbers. Dietary TC22 had no significant effects on total coelomocytes count, acid phosphatase activity, and superoxide dismutase activity in these animals $(P>0.05)$. Dietary TC22 at $10^{9} \quad \mathrm{CFU} / \mathrm{g}$ significantly improved phagocytosis, and total nitric oxide synthase activity in sea cucumbers $(P<0.05)$. Compared to the control, dietary TC22 had no significant effects on respiratory burst activity $(P>0.05)$. Respiratory burst in sea cucumbers fed dietary TC22 at $10^{9} \mathrm{CFU} / \mathrm{g}$ was significantly higher than those fed dietary TC22 at $10^{7} \mathrm{CFU} / \mathrm{g}$ $(P<0.05)$. Cumulative mortality after $V$. splendidus challenge decreased significantly in the sea cucumbers fed with TC22 at $10^{9} \mathrm{CFU} / \mathrm{g}(P<0.05)$. The present study confirmed dietary $B$. licheniformis TC22 at $10^{9} \mathrm{CFU} / \mathrm{g}$ could significantly improve immunity and disease resistance in juvenile $A$. japonicus.
\end{abstract}

\footnotetext{
* Corresponding author. e-mail: Yancui Zhao, E-mail address: yancuizh@126.com
} 


\section{Introduction}

Sea cucumber Apostichopus japonicus (Selenka) is one of the commercially important echinoderm species in China. The production of sea cucumbers in China, increased from 53,315 metric tons in 2004, to 200,969 metric tons in 2014 . However, the rapid increase in farming these sea cucumbers resulted in serious disease outbreaks such as skin ulceration syndrome, peristome tumescence, and viscera ejection syndrome (Wang et al., 2005). Although antibiotics are effective with some bacterial diseases in aquatic animals, their abuse has caused many problems (Yano et al., 2014). Hence many researchers have emphasized the importance of probiotics for farmed sea cucumbers (Liu et al., 2012; Chi et al., 2014; Li et al., 2015; Yang et al., 2015; Yang et al., 2016).

Probiotics in aquaculture are live, dead, or elements of microbic cells administered via feeds or to the rearing water and are advantageous to the host by increasing disease resistance, health, growth performance, feed optimization, stress and tolerance, responses (Ibrahem, 2015). The genus Bacillus is extensively used as a probiotic in aquaculture. It is known to improve growth, immunity, and disease resistance in sea cucumber (Zhao et al., 2012; Li et al., 2015; Yang et al., 2015; Zhao et al., 2015; Zhao et al., 2016). The use of autochthonous probiotics isolated from animals themselves or from their surroundings, including from sea cucumbers (Liu et al., 2012; Zhao et al., 2012; Chi et al., 2014; Yan et al., 2014; Li et al., 2015; Yang et al., 2015; Zhao et al., 2015; Yang et al., 2016; Zhao et al., 2016), and are more adaptable to their surroundings, and have a higher probability of success (De et al., 2014).

The present study was conducted to investigate the effects of a new autochthonous potential probiotic Bacillus licheniformis TC22 which was isolated from the intestines of infected sea cucumbers on growth, immunity, and disease resistance, against $V$. splendidus in $A$. japonicus (Selenka).

\section{Materials and methods}

Bacteria. The bacterial strain TC22 used in this study was isolated from the intestines of sea cucumbers infected by skin ulceration disease. It was identified as $B$. licheniformis by cluster analysis on the sequence of 16S rDNA (Ringø et al., 2006). Using the spot-on-lawn method (Pilet et al., 1995), TC22 was found to be inhibitory against $V$. splendius which caused this skin ulceration. The safety of TC22 was tested by immersing sea cucumbers in a suspension of TC22 at concentrations of $10^{3}, 10^{5}$, and $10^{7} \mathrm{CFU} / \mathrm{ml}$ (Verschuere et al., 2000). The safety test lasted 7 days. Every day the seawater was entirely renewed. After that, a fresh suspension of TC22 was added at final concentrations of $10^{3}, 10^{5}$, and $10^{7} \mathrm{CFU} / \mathrm{ml}$. Disease symptoms, and mortality of sea cucumbers were recorded every day. The safety test showed that TC22 did not induce disease symptoms and mortality of sea cucumbers.

Experimental diets. The powder of dried Sargassum thunbergii was used as basal feed. Experimental diets included supplemented graded doses of TC22 at $0,10^{5}, 10^{7}, 10^{9} \mathrm{CFU} / \mathrm{g}$ in the basal feed, respectively. S. thunbergii powder was sifted through a $149 \mu \mathrm{m}$ mesh. TC22 isolates were inoculated in trypticase soy broth (TSB) with $1.5 \% \mathrm{NaCl}$ and incubated for 48 $\mathrm{h}$ at $30^{\circ} \mathrm{C}$. They were then centrifuged at $5000 \mathrm{~g}$ for $10 \mathrm{~min}$ at $4^{\circ} \mathrm{C}$. After centrifugation, the bacteria were washed twice with sterile saline and their concentration was adjusted to $1 \times 10^{10} \mathrm{CFU} / \mathrm{ml}$ in sterile saline. Graded doses of TC22 were added into the S. thunbergii powder and mixed thoroughly. The experimental diets were freshly prepared every day to guarantee the vitality of TC22.

Feeding experiment. Juvenile sea cucumbers were obtained from a commercial farm in Qingdao, China. Prior to initiation of the experiment, the sea cucumbers were acclimated to the rearing conditions for 2 weeks. Then selected sea cucumbers of similar size $(0.204 \pm$ $0.009 \mathrm{~g}$, mean $\pm \mathrm{SE}$ ) were randomly distributed into 12 aquaria. Each aquarium ( $20 \mathrm{~L})$ was stocked with 40 sea cucumbers. Each diet was replicated in triplicate. During the 30 day feeding trial, all experimental animals were fed diets at the same fixed rate $(5 \%$ of body weight per day) twice daily (06:00 and 18:00), and 50\% of the water in each aquarium was replaced daily with fresh seawater. Water temperature was maintained at $20-22^{\circ} \mathrm{C}$, salinity 28-30, and $\mathrm{pH}$ 7.8-8.3. Low pressure electrical blowers provided aeration via air stones and maintained dissolved oxygen levels at or near air-saturation.

Sample collection. At the end of the feeding trial the sea cucumbers were fasted for $24 \mathrm{~h}$ and then dissected. The coelomic fluid was then collected immediately and thoroughly mixed with an equal volume of anticoagulant $(0.02 \mathrm{M}$ EGTA, $0.48 \mathrm{M} \mathrm{NaCl}, 0.019 \mathrm{M} \mathrm{KCl}, 0.068 \mathrm{M}$ Tris- 
$\mathrm{HCl}, \mathrm{pH}$ 7.6). Coelomic fluid from fifteen sea cucumbers from each aquarium was pooled for immunological analyses. After an aliquot was taken for total coelomocytes counts (TCC), phagocytosis activity test, and respiratory burst analysis, the rest of the anticoagulant coelomic fluid was centrifuged at $3000 \mathrm{~g}$ for $10 \mathrm{~min}$ at $4^{\circ} \mathrm{C}$ and resuspended in $600 \mu \mathrm{l}$ cold $0.85 \%$ saline, and sonicated at $22 \mathrm{kHz}$ for $25 \mathrm{~s}$ at $0^{\circ} \mathrm{C}$. This was centrifuged again at $4000 \mathrm{~g}$ for $10 \mathrm{~min}$ at $4^{\circ} \mathrm{C}$. After centrifugation, the cell lysate supernatant (CLS) was frozen in liquid nitrogen and stored at $-80^{\circ} \mathrm{C}$ for superoxide dismutase (SOD) activity, total nitric oxide synthase (T-NOS) activity, and acid phosphatase (ACP) activity assay.

Immune assays.

TCC. Coelomocytes were counted and calculated as cells per $\mathrm{ml}$ using a hemocytometer (Qiujing Inc., Shanghai, China) under light microscope at $400 \times$ magnification.

Phagocytic assay. Coelomocytes phagocytosis was evaluated following the method of Zhang et al. (2010). Three replicates of $100 \mu \mathrm{l}$ coelomic fluid of sea cucumbers from each aquarium were added to a 96 -wells plate and incubated at $25^{\circ} \mathrm{C}$ for $30 \mathrm{~min}$. The supernatant was removed and $100 \mu \mathrm{l} 0.029 \%$ neutral red (Shanghai, China) was added to each well. The plates were then incubated at $25^{\circ} \mathrm{C}$ for $30 \mathrm{~min}$. Cells were then washed with PBS 3 times and incubated with cell lysis buffer (acetic acid: ethanol $=1: 1$ ) for $20 \mathrm{~min}$. The results were recorded with a universal microplate spectrophotometer (Thermo, Waltham, MA, USA) using a test wavelength of $540 \mathrm{~nm}$. The absorbance of $10^{6}$ cells represents the capability of coelomocytes phagocytosing neutral red.

Respiratory burst activity. Production of superoxide anion was evaluated using nitroblue tetrazolium (NBT, Amresco, Solon, OH, USA) according to the method of Song and Hsieh (1994). Wells in a 96-well plate were coated with $100 \mu \mathrm{l} 0.2 \%$ poly-L-lysine (Sigma, St. Louis, MO, USA) solution to increase coelomocytes adhesion. Three replicates of $100 \mu \mathrm{l}$ aliquot of coelomic fluid from sea cucumbers in each aquarium were added to the wells and centrifuged at $300 \mathrm{~g}$ for $10 \mathrm{~min}$ at $4^{\circ} \mathrm{C}$. The supernatant was discarded and $100 \mu \mathrm{l}$ phorbol 1, 2-myristate 1, 3-acetate (PMA, Calbiochem, La Jolla, CA, USA) $(1 \mu \mathrm{g} / \mathrm{ml})$ was added to each well after which the plates were incubated at $37^{\circ} \mathrm{C}$ for $30 \mathrm{~min}$. The cells in each well were then stained with $100 \mu \mathrm{l} 0.3 \% \mathrm{NBT}$ at $37^{\circ} \mathrm{C}$ for $30 \mathrm{~min}$. Absolute methanol was added to terminate the staining. Each well was washed three times with $70 \%$ methanol and airdried. Then $120 \mu \mathrm{l} 2 \mathrm{M} \mathrm{KOH}$ and $140 \mu$ dimethyl sulfoxide (DMSO, Amresco, Solon, OH, USA) were added and the color was subsequently measured at $630 \mathrm{~nm}$ with a universal microplate spectrophotometer (Thermo, Waltham, MA, USA) using KOH/DMSO as a blank. The absorbance of $10^{6}$ cells represents the capability of respiratory burst activity in coelomocytes.

SOD activity. SOD activity was determined according to Ōyanagui (1984) with the assay kit of Nanjing Jiancheng, Bioengineering Institute, China. The reaction was based on its inhibitory effect on the rate of superoxide anion generated by xanthine and xanthine oxidase reaction system. The color was measured at $550 \mathrm{~nm}$ with a universal microplate spectrophotometer (Thermo, Waltham, MA, USA). One unit of SOD activity was defined as the amount of enzyme required for inhibiting superoxide-induced oxidation by $50 \%$. The specific SOD activity was expressed as SOD unit per mI CLS.

T-NOS activity. T-NOS activity was determined according to Green et al. (1982) with the assay kit of Nanjing Jiancheng, Bioengineering Institute, China. The reaction was based on its catalytic ability to convert L-Arginine into NO. NO was oxidized to nitrite $\left(\mathrm{NO}^{2-}\right)$ and nitrate $\left(\mathrm{NO}^{3-}\right)$. Nitrate $\left(\mathrm{NO}^{3-}\right)$ was converted to nitrite $\left(\mathrm{NO}^{2-}\right)$ utilizing nitrate reductase. Then the addition of Griess reagents converted all nitrite $\left(\mathrm{NO}^{2-}\right)$ into a color compound. Optical density was measured at $530 \mathrm{~nm}$ with a universal microplate spectrophotometer (Thermo, Waltham, MA, USA). One unit of T-NOS activity was defined as the amount of T-NOS producing $1 \mathrm{nmol} \mathrm{NO} / \mathrm{min}$. T-NOS specific activity was expressed as T-NOS unit per $\mathrm{ml}$ CLS.

ACP activity. ACP activity was determined by the method of King (1965) using disodium phenyl phosphate as substrate with a chemical detection kit (Nanjing Jiancheng, Bioengineering Institute, China). The unit definitions of ACP enzymatic activity corresponded to the degradation of $1 \mathrm{mg}$ phenol per $100 \mathrm{ml} \mathrm{CLS}$ at $37^{\circ} \mathrm{C}$ within 30 minutes.

A virulent strain of $V$. splendidus was provided by Yellow-sea Fishery Research Institute, Chinese Academy of Fishery Sciences (Qingdao, China). The strain was originally isolated 
from sea cucumbers infected by skin ulcerative syndrome (Zhang et al., 2006). The LD50 of sea cucumber tolerance to this virulent strain was determined prior to the challenge. LD50 for 7 days by immersion was $6 \times 10^{7} \mathrm{CFU} / \mathrm{ml}$. V. splendidus grew in TSB medium with $1.5 \%$ $\mathrm{NaCl}$ at $28^{\circ} \mathrm{C}$ for $24 \mathrm{~h}$. At the end of the feeding trial, 20 sea cucumbers from each aquarium were immersed with live $V$. splendidus at the final concentration of $6 \times 10^{7} \mathrm{CFU} / \mathrm{ml}$. The seawater was changed entirely every $24 \mathrm{~h}$ and fresh $V$. splendidus was again added into the aquarium. Mortality was monitored for 7 days.

Calculations and statistical analysis.

Specific growth rate $(\mathrm{SGR})=\left(\operatorname{Ln} W_{t}-\operatorname{Ln} W_{0}\right) \times 100 / t$

Where $W_{t}$ and $W_{o}$ were final and initial average individual weight of all sea cucumbers from each replicate respectively, and $t$ was duration of the experiment (30 days).

Statistical analysis was performed using the SPSS 16.0 for windows. The results are presented as mean \pm SE (standard error of the means). All percentage data were arcsine transformed. All data were subjected to one-way analysis of variance (ANOVA). When overall differences were significant at less than $5 \%$ level, Tukey's multiple range tests were used to compare the means between individual treatments.

\section{Results}

Growth performance. Final weight ranged from $0.523 \mathrm{~g}$ to $0.616 \mathrm{~g}$ (Table 1 ). SGR ranged from $2.361 \% / d$ to $3.491 \% / d$ (Table 1 ). SGR increased significantly $(P<0.05)$ in sea cucumbers fed with TC22 at $10^{9} \mathrm{CFU} / \mathrm{g}$ (Table 1 ).

Table 1. Effects of dietary TC22 on growth of sea cucumber Apostichopus japonicus after a 30-day feeding experiment (means $\pm \mathrm{SE}, \mathrm{n}=3)^{1}$

\begin{tabular}{cccc}
\hline $\log _{10}$ of TC22 & Initial weight $(g)$ & Final weight $(g)$ & Specific growth rate $(\% / d)$ \\
\hline 0 & $0.195 \pm 0.010$ & $0.523 \pm 0.031$ & $2.826 \pm 0.159^{\mathrm{b}}$ \\
5 & $0.237 \pm 0.018$ & $0.563 \pm 0.041$ & $2.475 \pm 0.068^{\mathrm{b}}$ \\
7 & $0.230 \pm 0.004$ & $0.526 \pm 0.021$ & $2.361 \pm 0.068^{\mathrm{b}}$ \\
9 & $0.183 \pm 0.019$ & $0.616 \pm 0.017$ & $3.491 \pm 0.227^{\mathrm{a}}$ \\
ANOVA & & & \\
$F$ Value & 3.534 & 2.203 & 11.995 \\
$P$ Value & 0.068 & 0.165 & 0.002 \\
\hline
\end{tabular}

1 Values was means and standard errors of three replicates;

2 ANOVA: Treatments with different letters are significantly different $(P<0.05)$.

Immune response.

TCC. TCC of different treatments ranged from $0.75 \times 10^{7} / \mathrm{ml}$ to $0.86 \times 10^{7} / \mathrm{ml}$. Compared to the control, dietary TC22 had no significant influence $(P>0.05)$ on the TCC of sea cucumbers (Fig. 1A).

Phagocytosis. Phagocytic activity of sea cucumbers significantly increased in sea cucumbers fed dietary TC22 supplements at $10^{5} \mathrm{CFU} / \mathrm{g}$ and $10^{9} \mathrm{CFU} / \mathrm{g}$ (Fig. 1B). Furthermore, phagocytic activity in the group with $10^{9} \mathrm{CFU} / \mathrm{g}$ dietary TC22 supplement was significant higher $(P<0.05)$ than those in groups with $10^{5} \mathrm{CFU} / \mathrm{g}$ and $10^{7} \mathrm{CFU} / \mathrm{g}$ dietary TC22 supplements (Fig. 1B).

Respiratory burst activity. There was no significant difference $(P>0.05)$ in respiratory burst activity of sea cucumbers fed diet containing TC22 compared with the control (Fig. 1C). However, respiratory burst activity of sea cucumbers fed diet containing TC22 at $10^{9}$ CFU/g was significantly higher $(P<0.05)$ than that of sea cucumbers fed diets containing TC22 at $10^{7}$ CFU/g (Fig. 1C).

Respiratory burst activity. There was no significant difference $(P>0.05)$ in respiratory burst activity of sea cucumbers fed diet containing TC22 compared with the control (Fig. 1C). However, respiratory burst activity of sea cucumbers fed diet containing TC22 at $10^{9}$ CFU/g was significantly higher $(P<0.05)$ than in sea cucumbers fed a diet containing TC22 at $10^{7} \mathrm{CFU} / \mathrm{g}$ (Fig. 1C).

SOD activity. Dietary TC22 at $10^{5}-10^{9} \mathrm{CFU} / \mathrm{g}$ had no significant influence $(P>0.05)$ on SOD activity in coelomocytes of sea cucumbers (Fig. 1D).

T-NOS activity. T-NOS activity of coelomocytes improved significantly $(P<0.05)$ in sea cucumbers fed dietary TC22 at $10^{9} \mathrm{CFU} / \mathrm{g}$ compared with those fed basal diet (Fig. 1E). Furthermore, the T-NOS activity of coelomocytes was higher in sea cucumbers fed dietary TC22 at $10^{5}$ and $10^{7} \mathrm{CFU} / \mathrm{g}$ than in those fed basal diet, although no significant difference $(P>0.05)$ was detected (Fig. 1E). 
ACP activity. The ACP activity in coelomocytes of sea cucumbers ranged from $3.83 \mathrm{U} / 100$ $\mathrm{ml}$ CLS to $4.72 \mathrm{U} / 100 \mathrm{ml}$ CLS. There was no significant difference $(P>0.05)$ in ACP activity of sea cucumbers fed with dietary TC22 at $0,10^{5}, 10^{7}, 10^{9} \mathrm{CFU} / \mathrm{g}$ (Fig. 1F).

$V$. splendidus immersion challenge
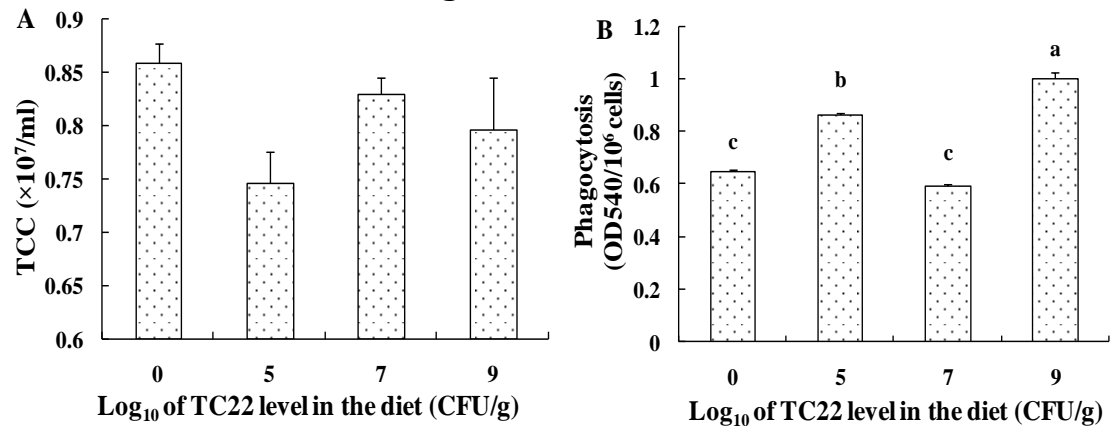

\section{Figure 1.}

Total coelomocytes count (TCC)

(A), phagocytosis (B), respiratory burst

(C), superoxide dismutase (SOD) activity

(D), total nitric oxide synthase (T-NOS) activity

(E), acid phosphatase

(ACP) activity

(F) juvenile
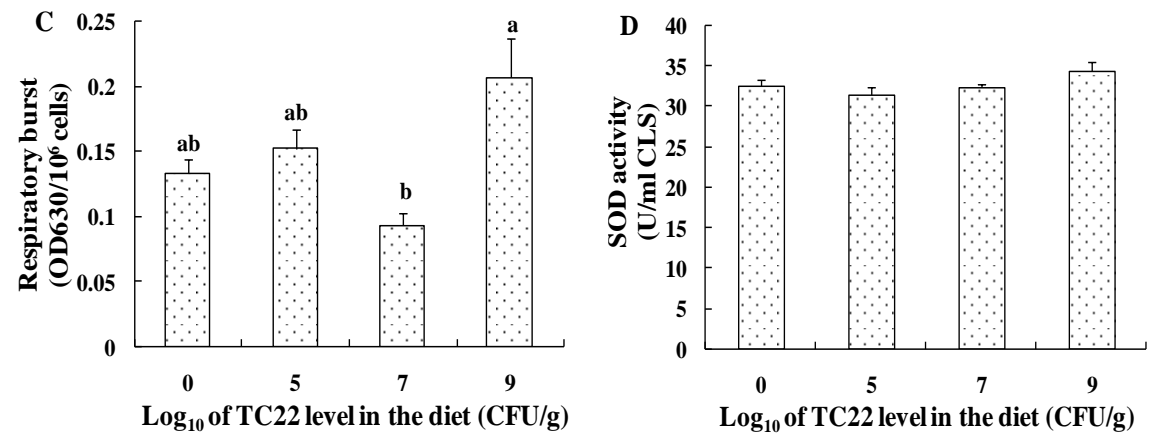

Apostichopus japonicus fed with graded doses of dietary TC22 for 30 days.

Values are means and standard errors of three replicates (means $\pm \mathrm{SE} ; \mathrm{n}=3$ ). Treatments with different letters are significantly different $(P<0.05)$.
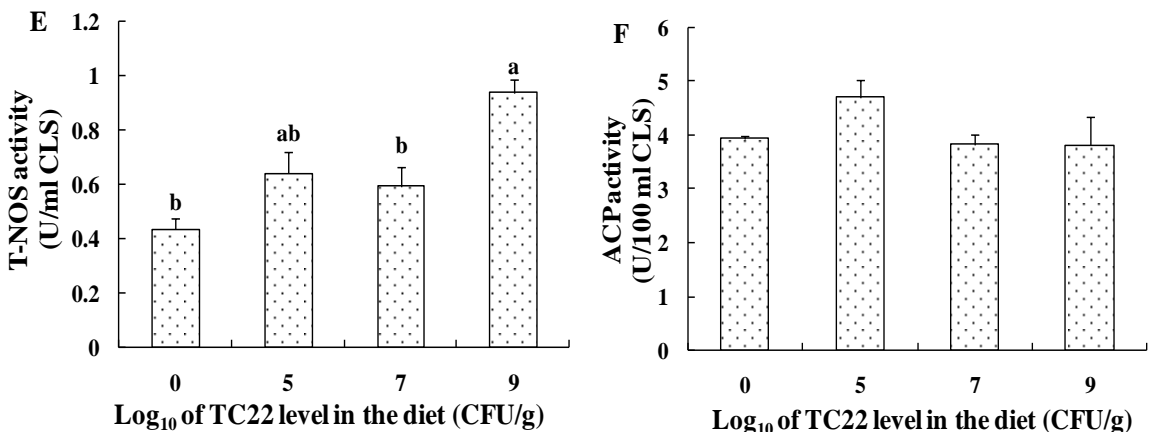

The immersion challenge test showed that administration of TC22 at $10^{9} \mathrm{CFU} / \mathrm{g}$ significantly $(P<0.05)$ enhanced disease resistance of sea cucumbers against $V$. splendidus infection (Fig. 2). However, there was no significant difference $(P>0.05)$ in the cumulative mortality rate of sea cucumbers fed with $10^{5} \mathrm{CFU} / \mathrm{g}$ or $10^{7} \mathrm{CFU} / \mathrm{g}$ dietary TC22 than that of sea cucumbers fed with basal diet. (Fig. 2).

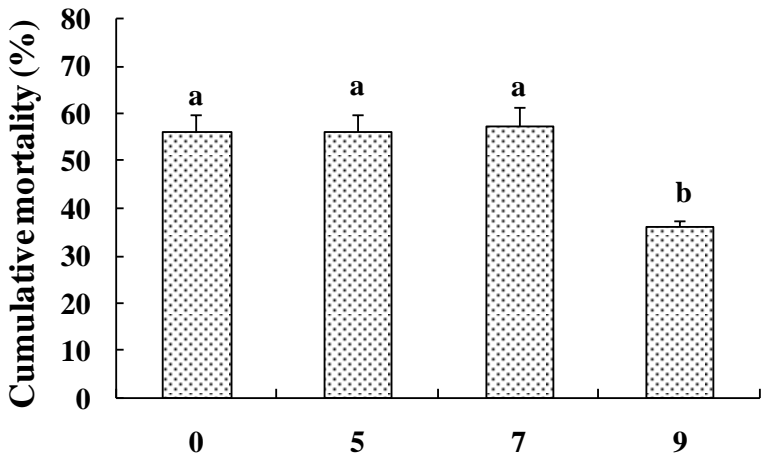

$\log _{10}$ of TC22 level in the diet (CFU/g)
Figure 2. Cumulative mortality during a 7 day $V$. splendidus challenge of juvenile sea cucumbers fed diets supplemented with graded levels of dietary TC22 for 30 days. Symbols represent means and standard errors of three replicate groups (means $\pm \mathrm{SE} ; \mathrm{n}=3$ ). Different letters indicate significant difference in mean cumulative mortality after a 7-day $V$. splendidus challenge $(P<0.05)$. 


\section{Discussion}

Growth performance of sea cucumbers was an important index used to evaluate the effects of probiotic Bacillus spp. on cultured sea cucumbers. Previous studies proved that at suitable doses, dietary Bacillus spp., such as indigenous B. subtilis T13 (Zhao et al., 2012), indigenous B. cereus (Yang et al., 2015; Tian et al., 2015), and commercial B. subtilis (Zhang et al., 2010) improved growth performance of sea cucumbers. Similarly, the present study showed that feeding $B$. licheniformis TC22 at $10^{9} \mathrm{CFU} / \mathrm{g}$ significantly increased the SGR of sea cucumbers. However, the precise mechanism by which Bacillus spp. increase the growth of sea cucumbers is still not clear. The present study showed that dietary $B$. licheniformis TC22 at $10^{9} \mathrm{CFU} / \mathrm{g}$ significantly increased innate immunity and disease resistance of sea cucumbers. The overall health of Sea cucumbers improved and they were able to gain more weight. Moreover, some previous studies confirmed that dietary Bacillus spp. at suitable doses could increase intestinal amylase and tripsin activity (Tian et al. 2015, Zhao et al. 2015), which may partially explain why growth of sea cucumbers was significantly increased by dietary Bacillus spp., i.e. higher amylase and tripsin activity promotes decomposition of carbohydrate and protein, and leads to improved digestion and absorption. It is therefore necessary to conduct further studies to elucidate the effects of $B$. licheniformis TC22 on the intestinal enzymes of sea cucumbers.

Coelomocytes of sea cucumbers recognize, engulf, and/or encapsulate invading microorganisms or foreign particles, and release humoral immune factors (Xing et al., 1998). Administration of $B$. licheniformis TC22 in the present study did not significantly influence TCC. This is in agreement with effects of dietary commercial probiotic product $B$. subtilis (Zhang et al., 2010), indigenous B. subtilis T13 (Zhao et al., 2012), indigenous probiotic mixture ( $B$. subtilis YB-1 and $B$. cereus YB-2) ( $\mathrm{Li}$ et al., 2015) and indigenous $B$. cereus EN25 (Zhao et al., 2016) on TCC of sea cucumbers. Many studies proved that oral administration of Bacillus spp. could improve the phagocytosis and respiratory bursts of coelomocytes in sea cucumbers (Zhang et al., 2010; Zhao et al., 2012; Li et al., 2015; Yang et al., 2015; Zhao et al., 2016). Similarly, TC22 was also found to increase phagocytosis in the present study. Although respiratory burst activity was not significantly increased by dietary B. licheniformis TC22, it was consistent with the variation of phagocytosis of coelomocytes in sea cucumbers. Phagocytosis and respiratory bursts of coelomocytes in the present study indicated that sea cucumbers fed TC22 at dosage of $10^{9} \mathrm{CFU} / \mathrm{g}$ were more resistant to microbial infections.

SOD catalyse the dismutation of extra bactericidal highly reactive $\mathrm{O}^{2-}$ to $\mathrm{O}_{2}$ and less reactive $\mathrm{H}_{2} \mathrm{O}_{2}$, and is an important enzyme in antioxidant defense system. The present study showed that dietary administration of $B$. licheniformis TC22 did not significantly influence SOD activity in coelomocytes of sea cucumbers. This result was similar to previous studies which found that feeding sea cucumbers dietary B. subtilis T13 (Zhao et al., 2012), $B$. cereus G19 (Yang et al., 2015), B. cereus BC-01(Yang et al., 2015) or a probiotic mixture ( $B$. subtilis $\mathrm{YB}-1$ and $B$. cereus $\mathrm{YB}-2$ ) ( $\mathrm{Li}$ et al., 2015) respectively had no significant influence on SOD activity. However, significantly increased SOD activity was found in sea cucumbers fed diets with Metschnikowia sp. C14 (Liu et al., 2012) and Paracoccus marcusii DB11 (Yan et al., 2014). Therefore, this difference could be partially attributed to the different genus of probiotics. In invertebrates, ACP plays an important role in the immune system for digestion of invading organisms. The present study indicated that dietary $B$. licheniformis TC22 did not significantly influence the immunity of sea cucumbers via ACP activity. This result was consistent with previous studies which demonstrated that dietary $B$. subtilis T13 (Zhao et al., 2012), dietary B. cereus G19 (Yang et al., 2015), B. cereus BC-01 (Yang et al., 2015) or dietary B. cereus EN25 (Zhao et al., 2016) had no significant influence on the ACP activities in sea cucumbers. Nitric oxide synthase (NOS) is responsible for production of NO, which is considered to be an important mediator in the protective immune response system (Wang et al., 2010). At present, there are only a few studies about the effects of probiotics on NOS activity of sea cucumber coelomocytes. Several probiotics such as $B$. subtilis T13 (Zhao et al., 2012), Metschnikowia sp. C14 (Liu et al., 2012) and $B$. cereus EN25 (Zhao et al., 2016) were found to significantly stimulate NOS activity in coelomocytes of sea cucumbers. Similar with these beneficial effects, the present study also demonstrated that dietary $B$. licheniformis TC22 at $10^{9} \mathrm{CFU} / \mathrm{g}$ significantly increased the NOS activity of coelomocytes in sea cucumbers. 
Dietary supplementation of Bacillus spp. strains including $B$. subtilis (Zhang et al., 2010; Zhao et al., 2012; Li et al., 2015), B. cereus (Li et al., 2015; Yang et al., 2015) and B. baekryungensis (Yan et al., 2014) increased disease resistance of sea cucumbers to pathogenic bacteria, such as $V$. splendidus and $V$. alginolyticus, through the promotion of growth and/or stimulation of celluar and humoral immune function. The present study also showed that the oral administration of $B$. licheniformis TC22 at $10^{9} \mathrm{CFU} / \mathrm{g}$ significantly reduced their cumulative mortality after challenged with $V$. splendius. This can possibly be explained on the basis of immunity stimulation of sea cucumbers induced by dietary $B$. licheniformis TC22 at $10^{\circ} \mathrm{CFU} / \mathrm{g}$ through increased phagocytosis, respiratory bursts and TNOS activity.

In conclusion, under the experimental conditions, dietary potential probiotic $B$. licheniformis TC22 at $10^{9} \mathrm{CFU} / \mathrm{g}$ can significantly increase growth, stimulate innate immunity, and increase resistance to skin ulceration disease caused by $V$. splendidus infection in juvenile sea cucumbers. The applications of $B$. licheniformis TC22 may present a novel strategy for health management in aquaculture of sea cucumbers.

\section{Acknowledgments}

The study was supported by National Natural Science Foundation of China (Grant No. 31202004), National High Technology Research and Development Program of China (863 Project, No. 2006AA100313), Ph.D. Programs Foundation of Lu Dong University (Grant No. LY2012009) and National Natural Science Foundation of China (Grant No. 31502173).

\section{References}

Chi, C., Liu, J.Y., Fei, S.Z., Zhang, C., Chang, Y.Q., Liu, X.L., Wang, G.X., 2014. Effect of intestinal autochthonous probiotics isolated from the gut of sea cucumber (Apostichopus japonicus) on immune response and growth of $A$. japonicus. Fish Shellfish Immunol., 38: 367-373.

De, B., Meena, D. ., Behera, B.K., Das, P., Das Mohapatra, P.K., Sharma, A.P., 2014. Probiotics in fish and shellfish culture: Immunomodulatory and ecophysiological responses. Fish Physiol. Biochem., 40: 921-971.

Green, L.C., Wagner, A., Glogowski, J., Skipper, P.L., Wishnok, J.S., Tannenbaum, S.R., 1982. Analysis of nitrate, nitrite, and $\left[{ }^{15} \mathrm{~N}\right]$ nitrate in biological fluids. Anal. Biochem., 126: $131-138$.

Ibrahem, M.D., 2015. Evolution of probiotics in aquatic world: Potential effects, the current status in Egypt and recent prospective. J. Adv. Res., 6: 765-791.

King, L., 1965. The hydrolases-acid and alkaline phosphatases. pp. 191-208. In: Van Nostrand, editor. Practical Clinical Enzymology, London, UK: Nostrand Company Limited.

Li, J.G., Xu, Y.P., Jin, L.J., Li X.Y., 2015. Effects of a probiotic mixture (Bacillus subtilis YB-1 and Bacillus cereus YB-2) on disease resistance and non-specific immunity of sea cucumber, Apostichopus japonicus (Selenka). Aquacult. Res., 46(12): 3008-3019.

Liu, Z.M., Ma, Y.X., Yang, Z.P., Li, M., Liu J., Bao, P.Y., 2012. Immune responses and disease resistance of the juvenile sea cucumber Apostichopus japonicus induced by Metschnikowia sp. C14. Aquaculture, 368-369: 10-18.

Ōyanagui, Y., 1984. Reevaluation of assay methods and establishment of kit for superoxide dismutase activity. Anal. Biochem., 142: 290-296.

Pilet, M.F., Dousset, X., Barre, R., Novel, G., Desmazeaud, M., Piard, J.C., 1995. Evidence of two bacteriocins produced by Carnobacterium piscicola and Carnobacterium divergens isolated from fish and active against Listeria monocytogene. J. Food Prot., 58: 256-262.

Ringø, E., Sperstad, S., Myklebust, R., Mayhew, T.M., Mjelde, A., Melle, W., Olsen R.E., 2006. The effect of dietary krill supplementation on epithelium-associated bacteria in the hindgut of Atlantic salmon (Salmo salar L.): a microbial and electron microscopical study. Aquac. Res., 37: 1644-1653.

Song, H.L., Hsieh, Y.T., 1994. Immunostimulation of tiger shrimp (Penaeus monodon) hemocytes for generation of microbicidal substances: analysis of reactive oxygen species. Dev. Comp. Immunol., 18: 201-209. 
Tian, X.L., Zhao, K., Wang, J., Yang, G., Yan, F.J., 2015. Influence of water additive and feed supplementary Bacillus cereus on the growth and activity of digestive and immune relating enzyme of sea cucumber. Periodical of Ocean University of China, 45(1): 18-25.

Verschuere, L., Rombaut, G., Sorgeloos, P., Verstraete, W., 2000. Probiotic bacteria as biological control agents in aquaculture. Microbiol. Mol. Biol. Rev., 64 (4): 655-671.

Wang Y.G., Xu, G.R., Zhang, C.Y., Sun S.F., 2005. Main diseases of cultured Apostichopus japonicus: prevention and treatment. Mar. Sci., 29: 1-7.

Wang, Y.J., Osatomi, K., Yoshida, A., Liang, X., Kanai, K., Oda, T., Hara K., 2010. Extracellular products from virulent strain of Edwardsiella tarda stimulate mouse macrophages (RAW264.7) to produce nitric oxide (NO) and tumor necrosis factor (TNF)-a. Fish Shellfish Immunol., 29: 778-785.

Xing, J., Leung, M.F., Chia, F.S., 1998. Quantitative analysis of phagcytosis by amoebocytes in a sea cucumber Holothuria leucospilota. Ivert. Biol., 117: 67-74.

Yan, F.J., Tian, X.L., Dong, S.L., Fang, Z.H., Yang, G., 2014. Growth performance, immune response, and disease resistance against Vibrio splendidus infection in juvenile sea cucumber Apostichopus japonicus fed a supplementary diet of the potential probiotic Paracoccus marcusii DB11. Aquaculture, 420-421: 105-111.

Yang, G., Tian, X.L., Dong, S.L., Peng, M., Wang, D.D., 2015. Effects of dietary Bacillus cereus G19, B. cereus BC-01, and Paracoccus marcusii DB11 supplementation on the growth, immune response, and expression of immune-related genes in coelomocytes and intestine of the sea cucumber (Apostichopus japonicus Selenka). Fish Shellfish Immunol., 45: 800-807.

Yang, H., Han, Y.Z., Ren, T.J., Jiang, Z.Q., Wang, F.Q., Zhang, Y.T., 2016. Effects of dietary heat-killed Lactobacillus plantarum L-137 (HK L-137) on the growth performance, digestive enzymes and selected non-specific immune responses in sea cucumber, Apostichopus japonicus Selenka. Aquacult. Res., 47(9): 2814-2824.

Yano, Y., Hamano, K., Satomi, M., Tsutsui, I., Ban, M., Aue-Umneoy, D., 2014. Prevalence and antimicrobial susceptibility of Vibrio species related to food safety isolated from shrimp cultured at inland ponds in Thailand. Food Control, 38(4): 30-36.

Zhang, C.Y., Wang, Y.G., Rong, X.J., 2006. Isolation and identification of causative pathogen for skin ulcerative syndrome in Apostichopus japonicus. J. Fish. China, 30: 118123.

Zhang, Q., Ma, H.M., Mai, K.S., Zhang, W.B., Liufu, Z.G., Xu, W., 2010. Interaction of dietary Bacillus subtilis and fructooligosaccharide on the growth performance, non-specific immunity of sea cucumber, Apostichopus japonicus. Fish Shellfish Immunol., 29: 204-211.

Zhao, K., Tian, X.L., Dong, S.L., Li, J., Wang, W.J., 2015. Effects of different states of Bacillus cereus on growth digestive enzyme activity and the immune defense factors of Apostichopus japonicus. Transactions of oceanology and limnology, 1: 63-72.

Zhao, Y.C., Yuan, L., Wan, J.L., Sun, Z.X., Wang, Y.Y., Sun, H.S., 2016. Effects of potential probiotic Bacillus cereus EN25 on growth, immunity and disease resistance of juvenile sea cucumber Apostichopus japonicus. Fish Shellfish Immunol., 49: 237-242.

Zhao, Y.C., Zhang, W.B., Xu, W., Mai, K.S., Zhang, Y.J., Liufu, Z.G., 2012. Effects of potential probiotic Bacillus subtilis T13 on growth, immunity and disease resistance against Vibrio splendidus infection in juvenile sea cucumber Apostichopus japonicus. Fish Shellfish Immunol., 32: 750-755. 